\title{
Magnetic resonance imaging assessment of reverse left ventricular remodeling late after restrictive mitral annuloplasty in early stages of dilated cardiomyopathy
}

Jos J. M. Westenberg, PhD, ${ }^{a, *}$ Jerry Braun, MD, ${ }^{b, *}$ Nico R. Van de Veire, MD, PhD, ${ }^{c}$ Robert J. M. Klautz, MD, PhD, ${ }^{b}$ Michel I. M. Versteegh, MD, ${ }^{b}$ Stijntje D. Roes, MD, ${ }^{d}$ Rob J. van der Geest, MSc, ${ }^{a}$ Albert de Roos, MD, PhD, ${ }^{d}$ Ernst E. van der Wall, MD, PhD, ${ }^{c}$ Johan H. C. Reiber, PhD, ${ }^{a}$ Jeroen J. Bax, MD, PhD, ${ }^{\mathrm{c}}$ and Robert A. E. Dion, MD, PhD ${ }^{\mathrm{b}}$

From the Department of Radiology, Division of Image Processing, ${ }^{\mathrm{a}}$ and Departments of Cardiothoracic Surgery, ${ }^{\mathrm{b}}$ Cardiology, and Radiology, ${ }^{\mathrm{d}}$ Leiden University Medical Center, The Netherlands.

* Westenberg and Braun both contributed equally to the study design, analysis, and article; shared first authorship is proposed.

Received for publication May 1, 2007; revisions received Sept 20, 2007; accepted for publication Oct 4, 2007.

Address for reprints: Jos J. M. Westenberg, $\mathrm{PhD}$, Division of Image Processing, Department of Radiology, Leiden University Medical Center, Albinusdreef 2, 2333 ZA Leiden, The Netherlands (E-mail: j.j.m. westenberg@lumc.nl).

J Thorac Cardiovasc Surg 2008;135:124753

\section{$0022-5223 / \$ 34.00$}

Copyright $\odot 2008$ by The American Association for Thoracic Surgery

doi:10.1016/j.jtcvs.2007.10.021
Objective: Magnetic resonance imaging was used to evaluate left ventricular reverse remodeling at long-term follow-up (3-4 years) after restrictive mitral annuloplasty in patients with early stages of nonischemic, dilated cardiomyopathy, and severe mitral regurgitation.

Methods: Twenty-two selected patients (eligible to undergo magnetic resonance imaging) with mild to moderate heart failure (mean New York Heart Association class $2.2 \pm 0.4$ ), dilated cardiomyopathy (left ventricular ejection fraction $37 \% \pm 5 \%$, left ventricular end-diastolic volume $215 \pm 34 \mathrm{~mL}$ ), and severe mitral regurgitation (grade 3-4+) underwent restrictive mitral annuloplasty. Magnetic resonance imaging was performed 1 week before surgery and repeated after 3 to 4 years.

Results: There was no hospital mortality or major morbidity. Two patients died during follow-up (9\%), and 2 patients could not undergo repeat magnetic resonance imaging because of comorbidity. New York Heart Association class improved from $2.2 \pm 0.4$ to $1.2 \pm 0.4(P<.05)$. Mitral regurgitation was minimal at late echocardiographic follow-up. There were significant decreases in indexed (to body surface area) left atrial end-systolic volume (from $84 \pm 20 \mathrm{~mL} / \mathrm{m}^{2}$ to $68 \pm 12 \mathrm{~mL} / \mathrm{m}^{2}, P<.01$ ), left ventricular end-systolic volume (from $42 \pm 14 \mathrm{~mL} / \mathrm{m}^{2}$ to $31 \pm 12 \mathrm{~mL} / \mathrm{m}^{2}, P<$ .01 ), left ventricular end-diastolic volume (from $110 \pm 18 \mathrm{~mL} / \mathrm{m}^{2}$ to $80 \pm 17 \mathrm{~mL}$ / $\mathrm{m}^{2}, P<.01$ ), and left ventricular mass (from $76 \pm 21 \mathrm{~g} / \mathrm{m}^{2}$ to $66 \pm 12 \mathrm{~g} / \mathrm{m}^{2}, P=$ $.03)$. Forward left ventricular ejection fraction improved from $37 \% \pm 5 \%$ to $55 \%$ $\pm 10 \%(P<.01)$. Indexed left atrial end-diastolic volume did not show a significant decrease (from $48 \pm 16 \mathrm{~mL} / \mathrm{m}^{2}$ to $44 \pm 10 \mathrm{~mL} / \mathrm{m}^{2}, P=.15$ ).

Conclusion: Magnetic resonance imaging confirms sustained significant reverse left atrial and ventricular remodeling at late (3-4 years) follow-up in patients with nonischemic, dilated cardiomyopathy, and mild to moderate heart failure after restrictive mitral annuloplasty.

$\mathrm{M}$ agnetic resonance imaging (MRI) is currently considered the gold standard for the assessment of left ventricular (LV) function and volumes. ${ }^{1}$ Advantages of MRI over echocardiography are the superior image quality and the 3-dimensional quantification possibilities with high reproducibility, implying that smaller sample sizes are needed to prove statistical significance of changes in LV volumes after therapy. ${ }^{2}$ MRI may therefore be the most appropriate imaging technique for the evaluation of surgical treatments for heart failure. In a previous study, we presented MRI data on short-term follow-up after restrictive annuloplasty in patients with nonischemic, dilated cardiomyopathy, and severe mitral regurgitation (MR). ${ }^{3}$ Significant left atrial (LA) and LV reverse remodeling were noted 2 months after surgery. Moreover, LV ejection fraction (LVEF) improved significantly. However, whether these beneficial effects are sustained at long-term follow-up is not clear. ${ }^{4}$ In the current study, the persistence of reverse remodeling at long-term follow-up after restrictive mitral annuloplasty is 


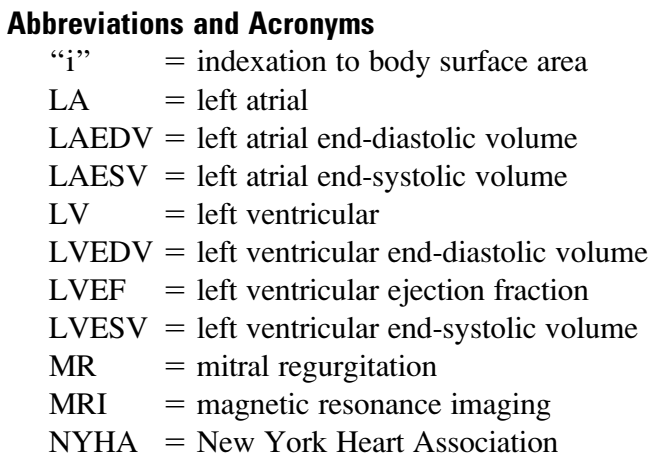

evaluated with MRI. Twenty-two selected patients with nonischemic, dilated cardiomyopathy, mild to moderate heart failure, and severe MR were evaluated by MRI within 1 week before restrictive mitral annuloplasty, and repeat imaging with MRI was performed at 3 to 4 years after surgery.

\section{Materials and Methods \\ Patients}

Twenty-two selected patients ( 18 male, 4 female, mean age $57 \pm 15$ years) with mild to moderate heart failure and dilated cardiomyopathy (New York Heart Association [NYHA] class $2.2 \pm 0.4, \mathrm{LV}$ end-diastolic dimension $61 \pm 5 \mathrm{~mm}$, forward LVEF $37 \% \pm 5 \%$, LV end-diastolic volume [LVEDV] $215 \pm 34 \mathrm{~mL}$ ) who were scheduled for isolated restrictive mitral annuloplasty were included. All patients presented with nonischemic, dilated cardiomyopathy (coronary artery disease excluded on coronary angiography) and severe functional MR on echocardiography. The patients had 3 to $4+$ MR (central jet) secondary to LV and annular dilatation and systolic restrictive motion of mitral leaflets (Carpentier type IIIb). All patients were receiving optimized medical therapy and kept on an optimal regimen during the study period.

Patients selected for this study had to be eligible to undergo repeat MRI examination. Therefore, in addition to general MRI exclusion criteria (ie, pacemakers/defibrillators, intracranial clips, pregnancy, claustrophobia), disease-related specific criteria were applied. These included the presence of (supra)ventricular arrhythmias or an existing indication for postoperative (biventricular) pacemaker/defibrillator implantation. To maintain uniformity of surgical intervention in this small patient group, additional valve surgery, including tricuspid valve repair, was another exclusion criterion. These factors inevitably limited the patient selection to those with relatively mild heart failure, a group of patients representing the better part of our heart failure program. None of the patients had pulmonary hypertension, and all patients had preserved right ventricular function. MRI was performed within 1 week before surgery and repeated 3 to 4 years later ( $43 \pm 8$ months). At follow-up, a routine transthoracic echocardiographic examination was performed.

\section{Surgery}

All surgical procedures were performed via a midline sternotomy under normothermic cardiopulmonary bypass with intermittent antegrade warm blood cardioplegia. The mitral valve was exposed through a vertical transseptal approach along the right border of the foramen ovale, leaving the roof of the left atrium untouched. Ring size (Carpentier-Edwards Physio ring, Edwards Lifesciences, Irving, Calif) was determined after careful measurement of the intercommissural distance and height of the anterior leaflet, and then downsizing by 2 ring sizes (ie, size 26 when measuring 30). All patients had intraoperative transesophageal echocardiographic assessment of valve function. Mitral valve repair was considered successful if there was no residual MR and a leaflet coaptation height of at least $8 \mathrm{~mm}$ at the A2-P2 level was achieved on intraoperative echocardiography.

\section{Magnetic Resonance Imaging}

MRI was performed using a 1.5 T MRI scanner (ACS-NT15 Gyroscan with the Powertrack 6000 gradient system; Philips Medical Systems, Best, The Netherlands). The body coil was used for transmission, and a 5-element phased-array synergy cardiac-coil was placed on the chest for signal reception. Standard 2- and 4-chamber long-axis series and a complete set of short-axis cine acquisitions were performed (conform standard cardiac MRI protocols ${ }^{5}$ using steady-state free precession ${ }^{6}$ ) with the patient performing a breath hold in end expiration. Imaging parameters of the 2- and 4-chamber long-axis series and for the short-axis series were as follows: TE/TR $=1.52 / 3.0$, flip angle $=50$ degrees, field of view $=350 \mathrm{~mm}$, scan matrix $=192 \times 153$, slice thickness $=8 \mathrm{~mm}$, and gated cardiac triggering with retrospective reconstruction of 30 phases. For the short-axis series, 10 to 12 parallel oriented slices were acquired with a 2-mm slice gap, 1 slice during each breath hold. LVEDV and LV end-systolic volume (LVESV) (from short-axis $\mathrm{MRI}^{7}$ ) and LAEDV and LAESV (from measuring biplane area-length in orthogonal long-axis 2- and 4-chamber views ${ }^{3}$ ) were obtained by manual segmentation. Image analysis was performed blinded with respect to echocardiographic data.

In the presence of significant MR, LVEF does not represent the true forward blood flow (through the aortic valve) because a substantial part of the blood volume leaks back into the left atrium. To correct for this effect, we have recently used the "forward LVEF," which was derived by calculating the ratio of the forward stroke volume and the EDV. ${ }^{3}$ The forward stroke volume was obtained from aortic flow measurements derived from velocity-encoded MRI. ${ }^{8}$ QMass and QFlow software (Medis, Leiden, The Netherlands) were used for image analysis. MRI examination was repeated at 3 to 4 years follow-up, and similar parameters were assessed. Significant reverse remodeling was defined as a volume reduction exceeding $15 \%$. An increase in forward LVEF of $5 \%$ or more and a decrease in LV mass $10 \mathrm{~g}$ or more were considered significant. ${ }^{3}$ The medical ethics committee of our institute approved all examinations. All patients gave informed consent.

\section{Statistical Analysis}

Continuous data were expressed as mean \pm standard deviation and compared using the Student $t$ test for paired data.

\section{Results}

\section{Clinical Outcome}

All patients underwent successful mitral valve repair. The median annuloplasty ring size was 26 . Intraoperative transesophageal echocardiography showed a mean coaptation 
length of $8 \pm 1 \mathrm{~mm}$. Residual MR was trivial in 4 patients and absent in the remaining patients. None of the patients had mitral stenosis, and systolic anterior movement of the anterior leaflet was not observed on echocardiography. There was no in-hospital or 30-day mortality, and no major complications occurred.

During follow-up, 2 patients (9\%) died of refractory heart failure before repeat MRI. In 2 additional patients, follow-up MRI could not be performed because of comorbidity (Alzheimer's disease and amyotrophic lateral sclerosis). There were no cases of endocarditis or thromboembolic events.

At late follow-up (43 \pm 8 months) NYHA class was $1.2 \pm$ $0.4(P<.05$ vs baseline), and transthoracic echocardiography showed minimal MR (mean grade $0.6 \pm 0.5$ ), with a mean leaflet coaptation length of $8 \pm 3 \mathrm{~mm}$, without mitral stenosis.
Reverse Remodeling on Magnetic Resonance Imaging Figure 1 shows the LA and LV reverse remodeling assessed by MRI. The MRI results are summarized in Table 1 . Although $\mathrm{LAEDV}_{\mathrm{i}}$ ( $\mathrm{i}$ ' indicating indexation to body surface area) did not decrease significantly (predefined by a volume reduction $\geq 15 \%$ ) (from $48 \pm 16 \mathrm{~mL} / \mathrm{m}^{2}$ to $44 \pm 10 \mathrm{~mL} / \mathrm{m}^{2}$, $P=.15$ ), significant LA reverse remodeling for EDV occurred in $56 \%$ of the patients. Individual data are presented in Figure 2, A. LAESV $\mathrm{i}_{\mathrm{i}}$ decreased significantly from $84 \pm 20$ $\mathrm{mL} / \mathrm{m}^{2}$ to $68 \pm 12 \mathrm{~mL} / \mathrm{m}^{2}(P<.01)$, with significant LA reverse remodeling for ESV occurring in $67 \%$ of patients (Figure 2, B).

LVEDV $_{\mathrm{i}}$ decreased significantly from $110 \pm 18 \mathrm{~mL} / \mathrm{m}^{2}$ to $80 \pm 17 \mathrm{~mL} / \mathrm{m}^{2}(P<.01)$. Significant reverse remodeling of LVEDV occurred in the majority of patients (89\%); individual data are shown in Figure 3, A. LVESV $\mathrm{i}_{\mathrm{i}}$ significantly
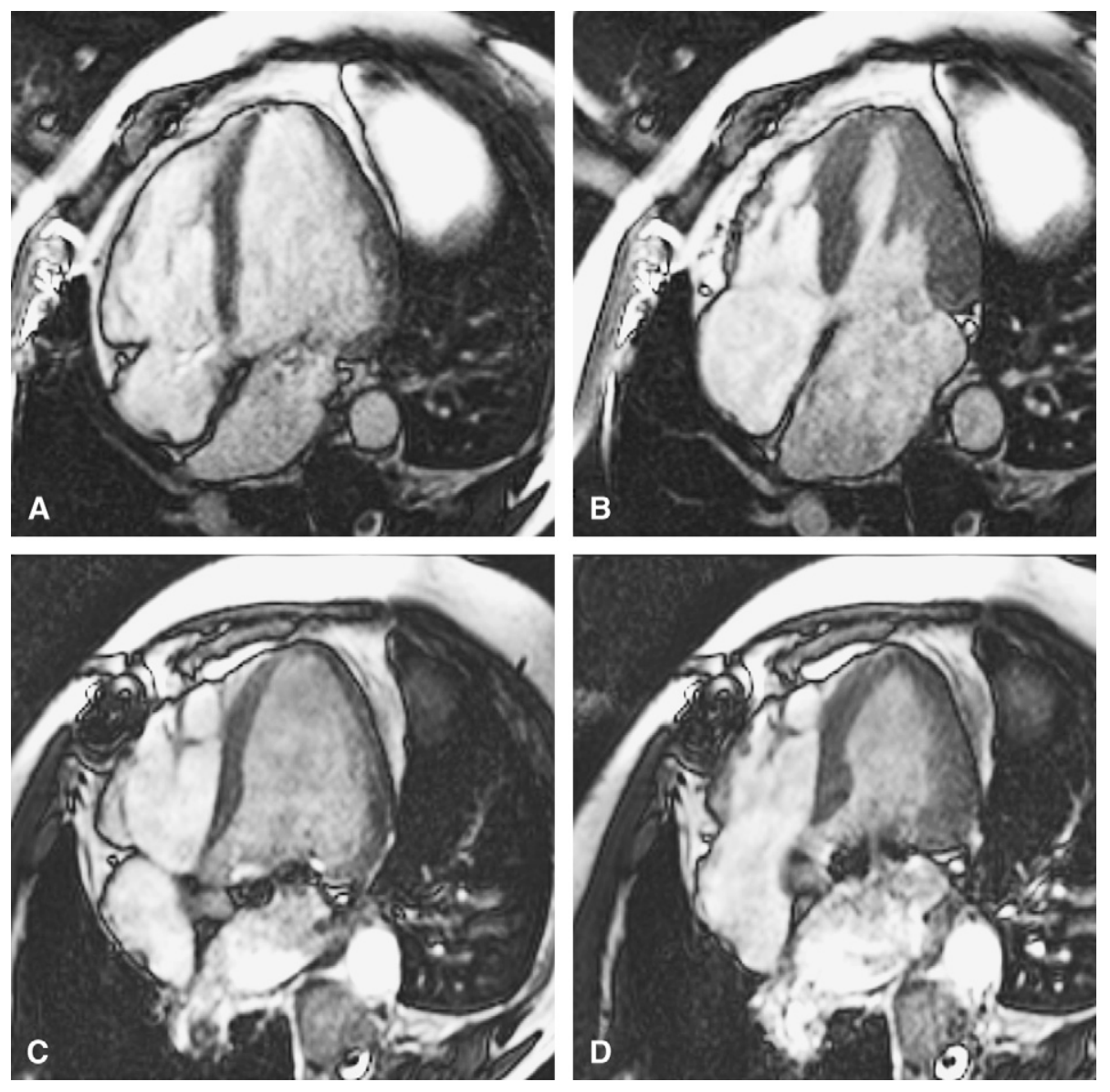

Figure 1. Patient example illustrating reverse remodeling of the left atrium and left ventricle 43 months after mitral valve repair. Four-chamber views during end diastole $(A)$ and end systole $(B)$ before surgery show severe dilatation of the left atrium (LAEDV $128 \mathrm{~mL}$, LAESV $216 \mathrm{~mL}$ ) and left ventricle (LVEDV $311 \mathrm{~mL}$, LVESV $90 \mathrm{~mL}$ ) caused by volume overload secondary to severe MR. The 4-chamber views during end diastole (C) and end systole (D), 43 months after surgery, show a reduction in LA volumes (LAEDV $88 \mathrm{~mL}$, LAESV $145 \mathrm{~mL}$ ) and LV volumes (LVEDV $210 \mathrm{~mL}$, LVESV $89 \mathrm{~mL}$ ). A restrictive semirigid ring is implanted in the mitral annulus, resulting in signal loss because of paramagnetic material inside the ring. 
TABLE 1. Magnetic resonance imaging results: Presurgery and at $43 \pm 8$ months follow-up

\begin{tabular}{|c|c|c|c|}
\hline & Presurgery & $43 \pm 8$ mo follow-up & $P$ value \\
\hline LAESV (mL) & $165 \pm 43$ & $132 \pm 20$ & $<.01$ \\
\hline LAESV $_{i}\left(\mathrm{~mL} / \mathrm{m}^{2}\right)$ & $84 \pm 20$ & $68 \pm 12$ & $<.01$ \\
\hline LAEDV $(\mathrm{mL})$ & $95 \pm 32$ & $85 \pm 19$ & .14 \\
\hline $\operatorname{LAEDV}_{\mathrm{i}}\left(\mathrm{mL} / \mathrm{m}^{2}\right)$ & $48 \pm 16$ & $44 \pm 10$ & .15 \\
\hline LVESV (mL) & $82 \pm 25$ & $60 \pm 22$ & $<.01$ \\
\hline $\operatorname{LVESV}_{i}\left(\mathrm{~mL} / \mathrm{m}^{2}\right)$ & $42 \pm 14$ & $31 \pm 12$ & $<.01$ \\
\hline LVEDV $(\mathrm{mL})$ & $215 \pm 34$ & $157 \pm 32$ & $<.01$ \\
\hline $\operatorname{LVEDV}_{\mathrm{i}}\left(\mathrm{mL} / \mathrm{m}^{2}\right)$ & $110 \pm 18$ & $80 \pm 17$ & $<.01$ \\
\hline Forward LVEF (\%) & $37 \pm 5$ & $55 \pm 10$ & $<.01$ \\
\hline LV mass/BSA $\left(\mathrm{g} / \mathrm{m}^{2}\right)$ & $76 \pm 21$ & $66 \pm 12$ & .03 \\
\hline
\end{tabular}

LAESV, Left atrial end-systolic volume; $L A E D V$, left atrial end-diastolic volume; $L V E S V$, left ventricular end-systolic volume; $L V E D V$, left ventricular end-diastolic volume; $L V E F$, left ventricular ejection fraction; $L V$, left ventricular; $B S A$, body surface area; $i$, indexation to body surface area.

decreased from $42 \pm 14 \mathrm{~mL} / \mathrm{m}^{2}$ to $31 \pm 12 \mathrm{~mL} / \mathrm{m}^{2}(P<.01)$, with $72 \%$ of patients showing significant reverse remodeling of LVESV (Figure 3, B). Overall, 2 patients (11\%) did not show any (LA or LV) reverse remodeling.

Forward LVEF increased significantly over time: from $37 \% \pm 5 \%$ at baseline to $55 \% \pm 10 \%(P<.01)$. Of note, $94 \%$ of patients showed a significant increase in forward LVEF (predefined as an increase of $\geq 5 \%$ in forward LVEF); individual results are shown in Figure 4, $A$. Indexed LV mass showed a statistically significant decrease (predefined by a mass reduction $\geq 10 \mathrm{~g}$ ) from $76 \pm 21 \mathrm{~g} / \mathrm{m}^{2}$ to $66 \pm 12 \mathrm{~g} / \mathrm{m}^{2}(P=.03)$. In addition, $83 \%$ of the patients showed a significant decrease in LV mass (Figure $4, B$ ).

\section{Discussion}

This study demonstrates the ability of MRI to report in a detailed fashion on various aspects of the LV reverse remodeling process (forward LVEF, LV and LA volumes).
All previous studies on improvement in LVEF and LV reverse remodeling after restrictive mitral repair in patients with dilated cardiomyopathy used echocardiography, which is limited by substantial intra- and interobserver variations and geometric assumptions needed for quantification of LVEF and LV volumes. The same measurements done with MRI have been reported to be highly accurate and reproducible. ${ }^{3,7}$ MRI has a superior image quality compared with echocardiography and is not hampered by technical limitations, such as suboptimal acoustic windows. Moreover, MRI has the advantage of acquiring every arbitrary double obliquely oriented imaging plane in 3 dimensions. Accordingly, MRI is considered the gold standard for assessment of LA and LV volumes, and its noninvasive nature and high reproducibility make this technique ideal for follow-up studies after therapy. ${ }^{9}$ The high reproducibility allows for smaller study sample sizes to prove statistical significance. For example, to demonstrate a $10-\mathrm{mL}$ difference in LVEDV with a $P$ value less than .05 and a power of $90 \%$, evaluation of only 12 patients is needed when MRI is used, compared with 121 patients when echocardiography is used. ${ }^{2}$ Indeed, in the current study, significant LA and LV reverse remodeling were statistically significantly proven with only 18 patients. Moreover, MRI permits easy correction for the influence of MR on LVEF by the use of the forward stroke volume (reflecting true forward blood flow through the aortic valve), as applied in the current study. This approach also permits a better appreciation of the true effect of mitral valve surgery on forward blood flow, reflected in the current study by a significant increase in forward LVEF.

However, the use of MRI for follow-up purposes is still limited by technical factors, and, as explained before, the patient group presented here represents the better part of our heart failure program. Currently, all patients with heart failure undergo MRI before surgery, but only those not requiring implantation of an epicardial LV lead or a pacemaker/defibrillator and those without (supra)ventricular arrhythmias are eligible to undergo follow-up MRI. These
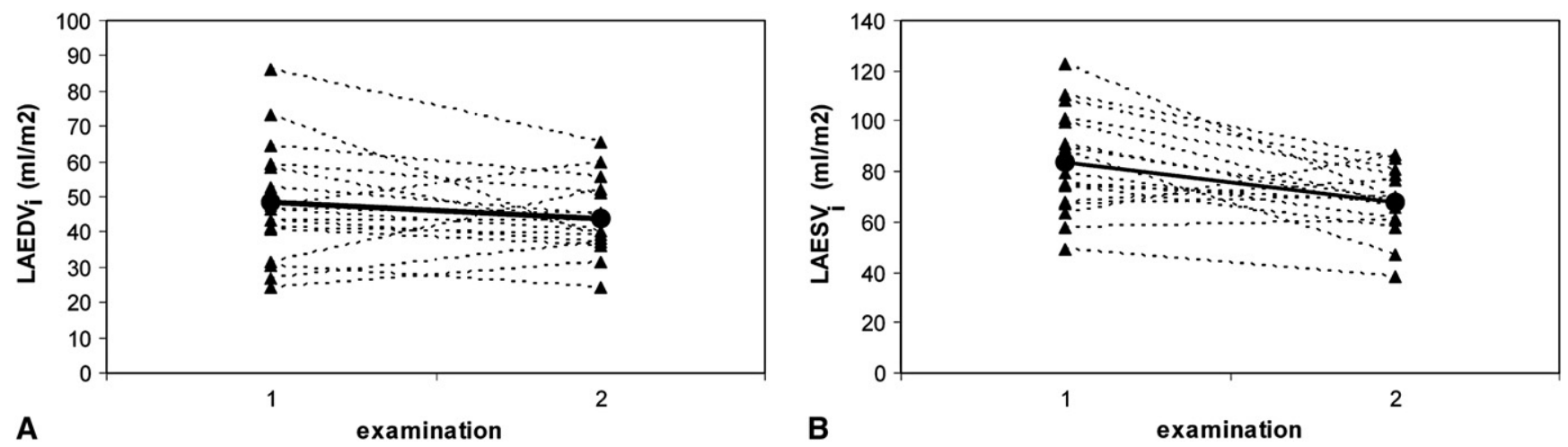

Figure 2. Individual data of patients demonstrating the changes over time in $\operatorname{LAEDV}_{i}(A)$ and LAESV $_{i}(B)$. Examination 1 concerns MRI before surgery; examination 2 concerns MRI at $43 \pm 8$ months follow-up. Mean values (circular data points and solid line); individual data (triangles and dotted lines). 

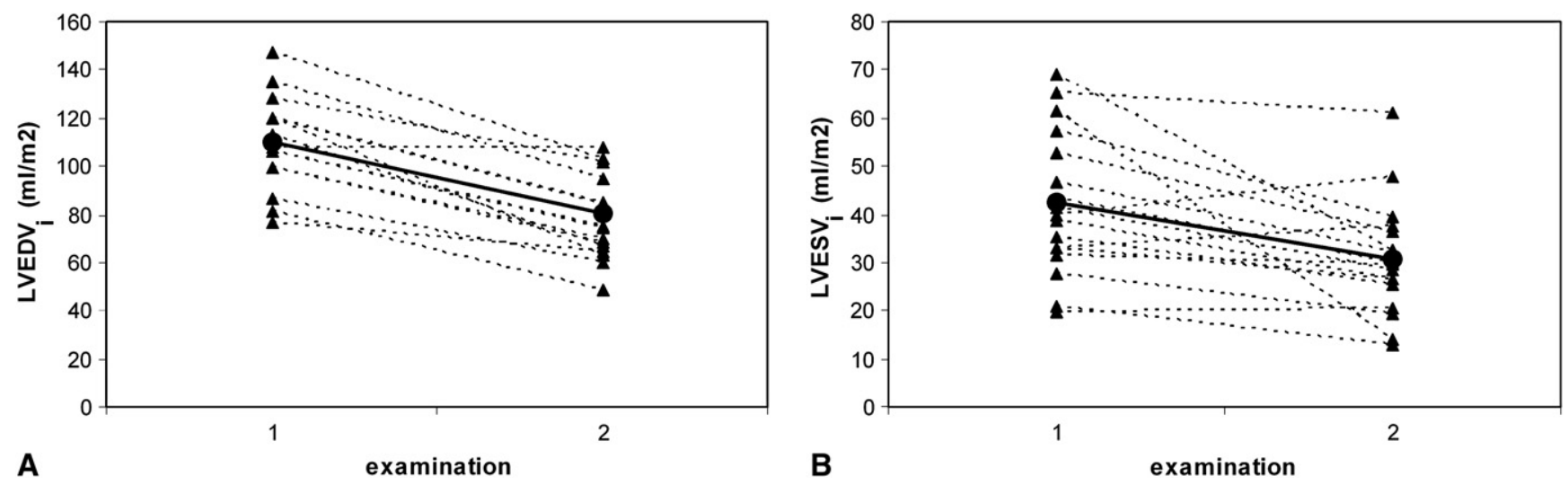

Figure 3. Individual data of patients demonstrating the changes over time in $\operatorname{LVEVV}_{i}(A)$ and $\operatorname{LVESV}_{i}(B)$. Examination 1 is MRI before surgery; examination 2 is MRI at $\mathbf{4 3} \pm \mathbf{8}$ months follow-up. Mean values (circular data points and solid line); individual data (triangles and dotted lines).

contraindications currently hamper widespread use of MRI in patients with heart failure. It is expected that these technical limitations of MRI will be overcome in the near future, which will greatly increase the possibilities of follow-up imaging in patients with heart failure.

In the current study, the beneficial effects of restrictive mitral annuloplasty in a selected group of patients with mild to moderate heart failure was shown. Currently, not much information on the effects of restrictive mitral annuloplasty in this patient category is available. Acker et $\mathrm{al}^{10}$ recently reported initial results from the Acorn trial. In this study, a subgroup of patients with mild heart failure (NYHA class II and relatively preserved LVEF and functional MR) underwent isolated mitral valve surgery, similar to the patients in the current study. The outcome of this subset of patients was not reported separately, making direct comparison with the current findings impossible.

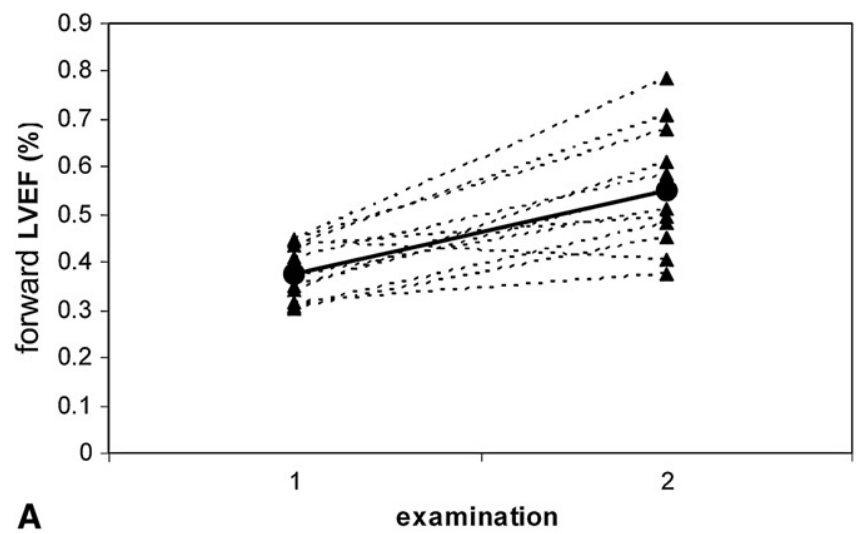

Another conclusion that can be drawn from the present study is that treatment of functional MR in nonischemic dilated cardiomyopathy by restrictive annuloplasty alone eliminates MR and leads to LA and LV reverse remodeling in the majority of this selected patient population. It has been questioned whether LV reverse remodeling is maintained over longer follow-up periods. Our current results confirm that $\mathrm{LV}$ reverse remodeling is indeed present at long-term follow-up. These favorable results may be related to the fact that only patients with milder heart failure were included (mean NYHA class $2.2 \pm 0.4$ and mean forward LVEF $37 \% \pm 5 \%$ ), reflecting the less ill patients in our heart failure program. In addition, only patients with preserved right ventricular function and without pulmonary hypertension or significant tricuspid regurgitation were selected. For reference, in our institution patients with more advanced stages of heart failure undergo tricuspid annuloplasty when significant tricuspid regurgitation or severe annular dilatation

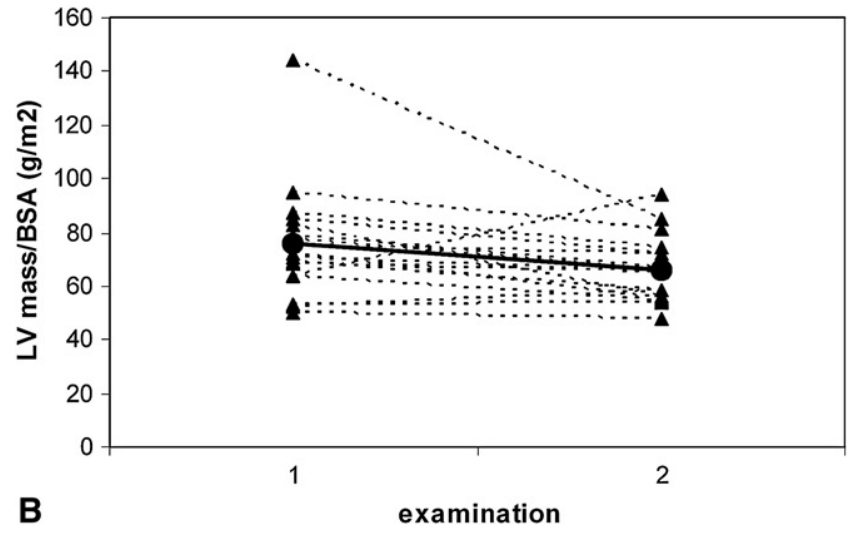

Figure 4. Individual data of patients demonstrating the changes over time in forward LVEF (A) and LV mass indexed by body surface area (B). Examination 1 is MRI before surgery; examination 2 is MRI at $43 \pm 8$ months follow-up. Mean values (circular data points and solid line); individual data (triangles and dotted lines). 
(annular diameter $\geq 40 \mathrm{~mm}$ ) is present. When LV enddiastolic diameter exceeds $65 \mathrm{~mm}$, an external cardiac constraint device (CorCap cardiac support device; Acorn Cardiovascular, St Paul, Minn) is also applied. These patients have a $6 \%$ operative mortality, and long-term results are currently being evaluated. When $\mathrm{LV}$ end-diastolic dimension exceeds $80 \mathrm{~mm}$, surgical LV restoration is considered as described by Isomura et al. ${ }^{11}$

The current results strengthen the hypothesis that grades 3 to 4+ functional MR can be successfully treated by restrictive mitral annuloplasty in patients with LV dilatation but relatively mild heart failure (NYHA class II to III). On a patient basis, $89 \%$ and $72 \%$ of patients exhibited a significant reduction in LV volumes. These observations are clinically relevant because LV function and volumes have been demonstrated to be important predictors of long-term outcome in patients with LV dysfunction. ${ }^{12,13}$ Also, LA dimensions have been shown to provide prognostic information. ${ }^{14,15}$ In the present study, LA volume in end systole showed a significant decrease, although LA volume in end diastole did not exhibit a decrease in the entire study population.

On the basis of the reverse remodeling observed in the current study, one could speculate that the point of no return (irreversible LV dilatation) was not yet reached in this patient category, suggesting that mitral valve surgery may be considered at an earlier stage in patients with nonischemic dilated cardiomyopathy. Further prospective studies are needed to confirm the benefit of early surgical intervention in patients with mild heart failure.

\section{Conclusions}

MRI provides a powerful tool for the evaluation of the LV and LA reverse remodeling process in patients with mild heart failure with functional MR and nonischemic dilated cardiomyopathy who underwent isolated restrictive annuloplasty. At late (3-4 years) follow-up, sustained significant LV and LA reverse remodeling was demonstrated using MRI. It should be emphasized that the current findings have been obtained in a highly selected patient population and that further MRI experience in patients with more advanced stages of heart failure is needed.

\section{References}

1. Van der Geest RJ, Reiber JHC. Quantification in cardiac MRI. J Magn Reson Imaging. 1999;10:602-8.

2. Bellenger NG, Davies LC, Francis JM, Coats AJ, Pennell DJ. Reduction in sample size for studies of remodeling in heart failure by the use of cardiovascular magnetic resonance. J Cardiovasc Magn Reson. 2000;2: 271-8.

3. Westenberg JJM, van der Geest RJ, Lamb HJ, Versteegh MIM, Braun J, Doornbos J, et al. MRI to evaluate left atrial and ventricular reverse remodeling after restrictive mitral annuloplasty in dilated cardiomyopathy. Circulation. 2005;112(9 Suppl):I437-42.

4. McGee EC, Gillinov AM, Blackstone EH, Rajeswaran J, Cohen G, Najam F, et al. Recurrent mitral regurgitation after annuloplasty for functional ischemic mitral regurgitation. J Thorac Cardiovasc Surg. 2004; 128:916-24.
5. Van Rossum AC, Visser FC, Van Eenige MJ, Valk J, Roos JP. Oblique views in magnetic resonance imaging of the heart by combined axial rotations. Acta Radiol. 1987;28:497-503.

6. Steenbeck J, Pruessmann K. Technical developments in cardiac MRI: 2000 update. Rays. 2001;26:15-34.

7. Pattynama PMT, Lamb HJ, van der Velde EA, van der Wall EE, de Roos A. Left ventricular measurements with cine and spin-echo MR imaging: a study of reproducibility with variance component analysis. Radiology. 1993;187:261-8.

8. Van der Geest RJ, Niezen RA, van der Wall EE, de Roos A, Reiber JHC. Automated measurement of volume flow in the ascending aorta using MR velocity maps: evaluation of inter- and intraobserver variability in healthy volunteers. J Comput Assist Tomogr. 1998;22:904-11.

9. Bellenger NG, Burgess MI, Ray SG, Lahiri A, Coats AJS, Cleland JGF, et al. Comparison of left ventricular ejection fraction and volumes in heart failure by echocardiography, radionuclide ventriculography and cardiovascular magnetic resonance; are they interchangeable? Eur Heart J. 2000;21:1387-96.

10. Acker MA, Bolling S, Shemin R, Kirklin J, Oh JK, Mann DL, et al. Acorn Trial Principal Investigators and Study Coordinators. Mitral valve surgery in heart failure: insights from the Acorn Clinical Trial. J Thorac Cardiovasc Surg. 2006;132:568-77.

11. Isomura T, Suma H, Hori T, Sato T, Kobashi T, Kanemitsu H, et al. Left ventricular restoration in patients with non-ischemic dilated cardiomyopathy: risk factors and predictors of outcome and change of mid-term ventricular function. Eur J Cardiothorac Surg. 2001;19:684-9.

12. Udelson JE, Konstam MA. Relation between left ventricular remodeling and clinical outcomes in heart failure patients with left ventricular systolic dysfunction. J Card Fail. 2002;8(Suppl):S465-71.

13. White HD, Norris RM, Brown MA, Brandt PWT, Whitlock RML, Wild CJ. Left ventricular end-systolic volume as the major determinant of survival after recovery from myocardial infarction. Circulation. 1987; $76: 44-51$

14. Bax JJ, Braun J, Somer ST, Klautz R, Holman ER, Versteegh MI, et al. Restrictive annuloplasty and coronary revascularization in ischemic mitral regurgitation results in reverse left ventricular remodeling. Circulation. 2004;110(Suppl 1):II103-8.

15. Dini FL, Cortigiani L, Baldini U, Boni A, Nuti R, Barsotti L, et al. Prognostic value of LA enlargement in patients with idiopathic dilated cardiomyopathy and ischemic cardiomyopathy. Am J Cardiol. 2002;89: $518-23$.

\section{Discussion}

Dr Michael Acker (Philadelphia, $\mathrm{Pa}$ ). I congratulate the authors for this well-conducted study using what is considered by many as the gold standard in looking at cardiac volumes. You confirmed in your patients with moderate heart failure that by correcting MR we can see reverse remodeling, which we have seen in many other types of groups. I have 4 questions.

Mitral insufficiency and heart failure are often very dynamic, and these patients were not that sick. Were these patients really optimized on standard advanced medical therapy before you attempted to operate? How long were they followed before making the decision to operate?

Dr Jerry Braun (Leiden, The Netherlands). All patients were on a good medical treatment strategy. All patients had at least had diuretics.

Dr Acker. Were they all on beta-blockers and angiotensinconverting enzyme inhibitors? If not, what percentage?

Dr Braun. All patients were receiving angiotensin-converting enzyme inhibitors, and the majority were receiving beta-blockers ( $~ 80 \%$ or $90 \%$ ), this was continued after surgery.

Dr Acker. I saw that there was a bit of residual MR at your followup $(0.6 \pm)$, suggesting that some patients had up to $2+$ MR. If you ever 
do see that, can you give us a hint on what preoperative characteristics of the patients, the ventricle, or the actual mitral valve might indicate late failure of your repair with a small annuloplasty ring?

Dr Braun. In this group, none of the patients had grade 2 MR. We know from our studies in ischemic patients, after 4 years about $85 \%$ has no grade $2 \mathrm{MR}$, while $15 \%$ has. In that patient group we tried to relate it to preoperative variables, but were not able to find a relation. It occurs both in the larger ventricle group as well as in the smaller ventricle group.

Dr Acker. Nothing about the degree of coaptation depth or annular size or whether it's an eccentric jet or central jet would indicate?

Dr Braun. No, not at all.

Dr Acker. Two of your patients died. At death-I don't know if they were censored from your results or not-did they have return of MR and did you see a lack of reverse remodeling in these 2 patients who died of progressive heart failure?

Dr Braun. Two patients died. One patient died several months after surgery, suddenly, probably because of ventricular arrhythmias. The other died shortly after emergent surgery for a vascular problem. The last echo several months before death showed a competent mitral valve and no progression of LV dilatation.

Dr Acker. You've demonstrated that there is reverse modeling in moderate heart failure. Do you have any evidence that you can share with us for patients with advanced heart failure secondary to idiopathic myopathy, such as these, and for patients with ischemic cardiomyopathy?
Dr Braun. We're working on our complete series of nonischemic patients. Unfortunately, we're not able to follow them with MRI, but with echocardiography. We're still working on that. I should say that currently we treat these patients with the CorCap cardiac support device (Acorn Cardiovascular; St. Paul, Minn, USA) device if they have an LV end-diastolic dimension exceeding $65 \mathrm{~mm}$; we don't only do a restrictive mitral annuloplasty.

I should say that we treat these patients currently, if they have a left ventricular end diastolic dimension exceeding $65 \mathrm{~mm}$, not only by a restrictive mitral annuloplasty, but we also add a CorCap cardiac support device (Acorn Cardiovascular; St. Paul, Minn, USA). In the ischemic patients, that we presented at the STS meeting in San Diego earlier this year we obtained results that are quite promising, as I said. Maybe I can show a discussion slide.

Dr Starnes. I don't think we have time.

Dr Braun. The results are very promising.

Dr Hormoz Azar (Norfolk, $\mathrm{Va}$ ). There will be a certain amount of remodeling just by inserting the ring. Did you measure the midventricular diameter and did that show diminution in time, or how much of it was related just to cinching the ventricle by the ring?

Dr Braun. Of course, that difference is difficult to make. We measured the volumes in this series, so we get an overall impression of the ventricle. By echocardiography, we measure the end-diastolic dimension at the level where the tips of the mitral leaflets close, or at least where the tips of the papillary muscles are located, and we see a decrease at that level as well. But that is not a way to discern whether this is why MR is observed. 